\title{
What the tortoise never said to Achilles*
}

\author{
Oliver Penrose
}

ACHILLES had overtaken the Tortoise, and had seated himself comfortably on its back. "So you've got to the end of our race - er, our book" said the Tortoise. "Even though it DOES consist of an infinite variety of self-referring statements, curious puns, paradoxes, and speculations? I thought some wiseacre or other had proved that the thing couldn't be done?"

"It can be done", said Achilles. "It HAS been done! Solvitur legendo. You see each pun or paradox only took HALF as long to read as the one before; and so ..."

"But the Index", interrupted the Tortoise, "how did you finish THAT? Every time you came to the entry 'Achilles: mapped on to ant colony 318,324 ' you surely had to turn back to the middle of the book, so how could you EVER finish?"'

"That splendid index", murmured Achilles. "It's true I did have some trouble getting past the entry 'Zen Buddhism; computers and, 625 - 26' especially as one of the chapters is based on Zen kóans, but even so I'm sure I'd have beaten you if I hadn't spent so long trying to do that fascinating MIU puzzle in chapter 1"'.

"Oh yes", recalled the Tortoise, "given the string of letters MI, and the rules for producing new strings:

1. If your string ends with $I$, you can add a $U$ at the end.

2 . If your string is $M x$, where $x$ is any string, you can change it to $\mathrm{Mxx}$.

3. If your string contains the sequence III, you can replace that sequence by $U$.

4. If your string contains the sequence UU, you can delete that sequence.

can you produce the string MU?"

"Well, I'm only a simple warrior", said Achilles "and I never did manage to produce MU, but I thought the puzzle was a BRILLIANT way of introducing the idea of a formal logical system."

"And formal logical systems are very important", said the Tortoise, "because the centrepiece of the book is a sketch of the proof of Gödel's theorem: that practically any formal logical system contains statements which can't be either proved or disproved within the system."

"Ah yes, that proof of Godel's theorem", sighed Achilles. "Very entertaining, like the rest of the book, but even after reading it an INFINITE number of times I still don't think I understand it.",

"With apologies to Lewis Carroll "What the Tortoise said to Achilles", Mind, n.s.4 (1895), pp.278-80, reprinted in pp.43-45 of the book reviewed here.
Godel, Escher and Bach: An Eternal Golden Braid - A Metaphorical Fugue on minds and machines in the spirit of Lewis Carroll. By D. R. Hofstadter. Pp.777. (Basic Books: New York, 1978; Harvester: Brighton, UK, 1979.) \$18.50; $£ 10.50$.

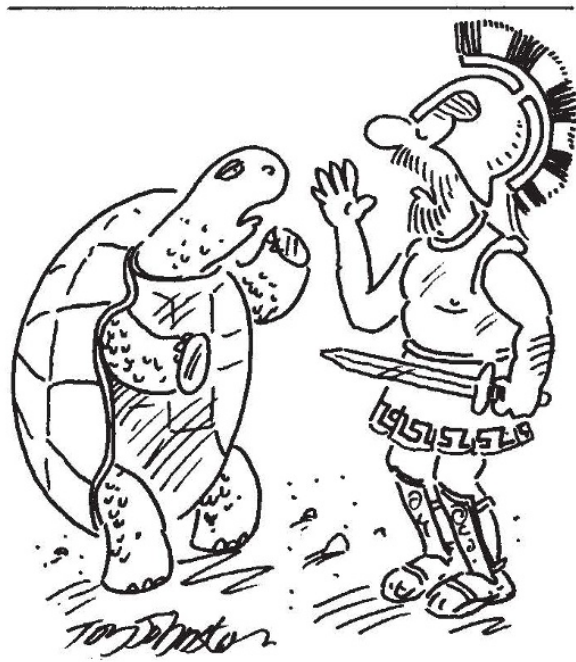

"Each reading taking only half as long as the previous one, of course", mused the Tortoise. "But I think the author's intention was not so much to give a crystalclear explanation of Gödel's proof (he might possibly have done that better by making more use of Cantor's diagonal construction) as to exploit the idea of selfreference to the full. So he gives, as an analogue of Gödel's undecidable formal proposition, the following English statement: "yields falsehood when preceded by its quotation' yields falsehood when preceded by its quotation."

"That must be the grandmother of all confusing self-referring sentences", said Achilles, mopping his brow. "I never DID manage to decide whether that one is true or not. But rather than get worried about it I turned to the Escher lithographs which he uses so delightfully to illustrate many of his ideas - for example, the picture of someone in an art gallery looking at a picture of a town which contains an art gallery which contains ...".

"Do you mind", interrupted the Tortoise, "you are sticking your sword into my foot."

"MIND?", said Achilles, waving the sword excitedly. "That's just the kind of thing the rest of the book is about. What is a mind? Where is meaning located? How is knowledge represented? Can a computer be conscious? Can animals with very primitive nervous systems be said to think? ...'

"More easily than featherbrained warriors", muttered the Tortoise testily.

"Do you remember the description in the book of Terry Winograd's artificial intelligence device SHRDLU," went on Achilles, as if the Tortoise had not spoken, "which can move solid objects around in response to commands like "find a block which is taller than the one you are holding and put it into the box'?"

"With comments by "the newly developed human being, Dr Tony Earrwig' who seems to be related in a mixed-up way to SHRDLU's creator" added the Tortoise. "By the way, if you had to read the chapter about Gödel's theorem so often, I'm amazed that you finished the book so quickly."

"Well, I have to admit", said Achilles, "that to help me catch up with you, I only read every other chapter. The book still seemed to make sense though."

"What?" The Tortoise was horrified. "You missed the best part. Those chapters are all about US. And you missed my wonderful invention, the Tortoise property, enjoyed by every number that is the difference of two primes".

"Are you sure you don't mean the Goldbach property, which is that the number is the suM of two primes?"

"No, the difference. The Goldbach property is VERY dull: you can always tell in a finite number of steps whether a given number has it or not, whereas if a number does not have the Tortoise property you could go on trying primes for ever. Besides that", continued the Tortoise, "you missed some extremely ingenious dialogues, which are verbal imitations of contrapuntal devices used by Bach in his much-loved works such as the Musical Offering. They provide welcome points of relaxation between the more serious chapters that you did read."

"With Bach as a model, the book must be even better constructed than I thought", said Achilles. "And the author's enthusiasm certainly carries you along as you read. But don't I see our mixed-up relatives Les Cahil and Sir Otto E. over there? Let's go and join them".

Oliver Penrose is a Professor of Mathematics at the Open University, Milton Keynes, UK. 